\title{
Diagnosis of an aneurysmal bone cyst of the cricoid cartilage: A case report
}

\author{
YUSHENG CHENG $^{1 *}$, HONGMING XU ${ }^{1 *}$, ANTON A. KVIT ${ }^{2}$ and ZIWEI YU ${ }^{1}$ \\ ${ }^{1}$ Department of Otolaryngology, Head and Neck Surgery, Shanghai Jiao Tong University Affiliated First People's Hospital, \\ Shanghai 200080, P.R. China; ${ }^{2}$ Department of Surgery, University of Wisconsin, Madison, WI 53562, USA
}

Received November 4, 2014; Accepted April 28, 2015

DOI: $10.3892 / \mathrm{etm} .2015 .2513$

\begin{abstract}
An aneurysmal bone cyst (ABC) is a type of reactive reparative bone neoplasm that rarely occurs in the head and neck. To date, only a small number of cases have been previously reported. The present study reports the case of a 58-year-old male who presented with a tumor in the laryngeal cricoid cartilage, a rare position for a tumor, who subsequently underwent treatment by surgical resection. Based on the observations of the present study and a literature review, it was concluded that an ABC of the larynx can be identified based on a combination of radiology and postoperative histopathology examinations. However, following a tracheotomy and excision, the difficulty of performing tracheal cannula removal is inevitable. The present study described in detail a rare disease, $\mathrm{ABC}$, occurring in the larynx. More cases are required for follow-up studies.
\end{abstract}

\section{Introduction}

The cricoid cartilage is the only ring-like cartilage of the human larynx, which partially supports the laryngeal cavity and prevents the respiratory tract from becoming blocked. To the best of our knowledge, an aneurysmal bone cyst (ABC) is a rare disease with the earliest case reported in 1986 (1). Only 4 cases were found in the area of otorhinolaryngology, head and neck surgery. An ABC is a locally aggressive, but benign bony tumor (2). The most recent diagnosis was based on pathological diagnosis although the microscopic features of an $\mathrm{ABC}$ are characterized by uncoagulated blood, in addition to a certain extent of bone and bone-like fragments. Through

Correspondence to: Professor Ziwei Yu, Department of Otolaryngology, Head and Neck Surgery, Shanghai Jiao Tong University Affiliated First People's Hospital, 100 Haining Road, Shanghai 200080, P.R. China

E-mail: david545yu@yahoo.com

${ }^{*}$ Contributed equally

Key words: pathological mechanism, aneurysmal bone cyst of the cricoid cartilage, magnetic resonance imaging, surgical treatment analyzing the observations of previous studies, the imaging manifestations of an $\mathrm{ABC}$ were determined to have a high clinical significance. The main treatment methods for ABCs include excision, curettage and hormone injection therapy, however, only one case has been successfully cured by simple excision with voice preservation (3). The current study presents the case of a 58-year-old male patient with an ABC located in the cricoid cartilage.

\section{Case report}

A 58-year-old male patient was admitted to Shanghai Jiao Tong University Affiliated First People's Hospital (Shanghai, China) complaining of shortness of breath that had lasted for $>6$ months. In addition, the patient had presented with progressively troubled breathing accompanied with intermittent throat soreness for more than half a year previously, without evident triggers. At that point in time, the symptoms were eased following the administration of antibiotic treatment, including intravenous infusion of $2.0 \mathrm{~g}$ cefradine twice a day for five consecutive days. At one week prior to hospital admission, the patient experienced dyspnea, which was slightly relieved by adopting a sitting-up position. No voice changes were recorded and the past medical history of the patient was unremarkable. Furthermore, the patient had no previous history of respiratory disease or trauma to the neck, and the physical examination provided no new diagnostic clues. Written informed consent was obtained from the patient. Relevant laboratory examinations, including the serum electrolyte level and a complete blood count, were within normal limits. In addition, routine radiographs of the chest revealed no significant abnormality. However, a laryngoscopy revealed that the postcricoid area was humped and the mucosal surface was edematous. Magnetic resonance imaging (MRI) of the larynx, following hospital admission, revealed an irregular soft tissue mass that grew forward and compressed the trachea in the postcricoid area (Fig. 1A). The mass had a vertical diameter of $\sim 18 \mathrm{~mm}$ and maximum cross-section measuring $36 \mathrm{~mm}$ by $20 \mathrm{~mm}$. The upper edge of the cyst was shown to reach the submandibular gland (Fig. 1B). In addition, T1-weighted imaging revealed the mass to be fuzzy with a slightly higher signal compared with the muscle signal (Fig. 2A), while the T2-weighted images showed the mass had an uneven cavity with a high signal, and a weak signal line between the sac intervals (Fig. 2B). 

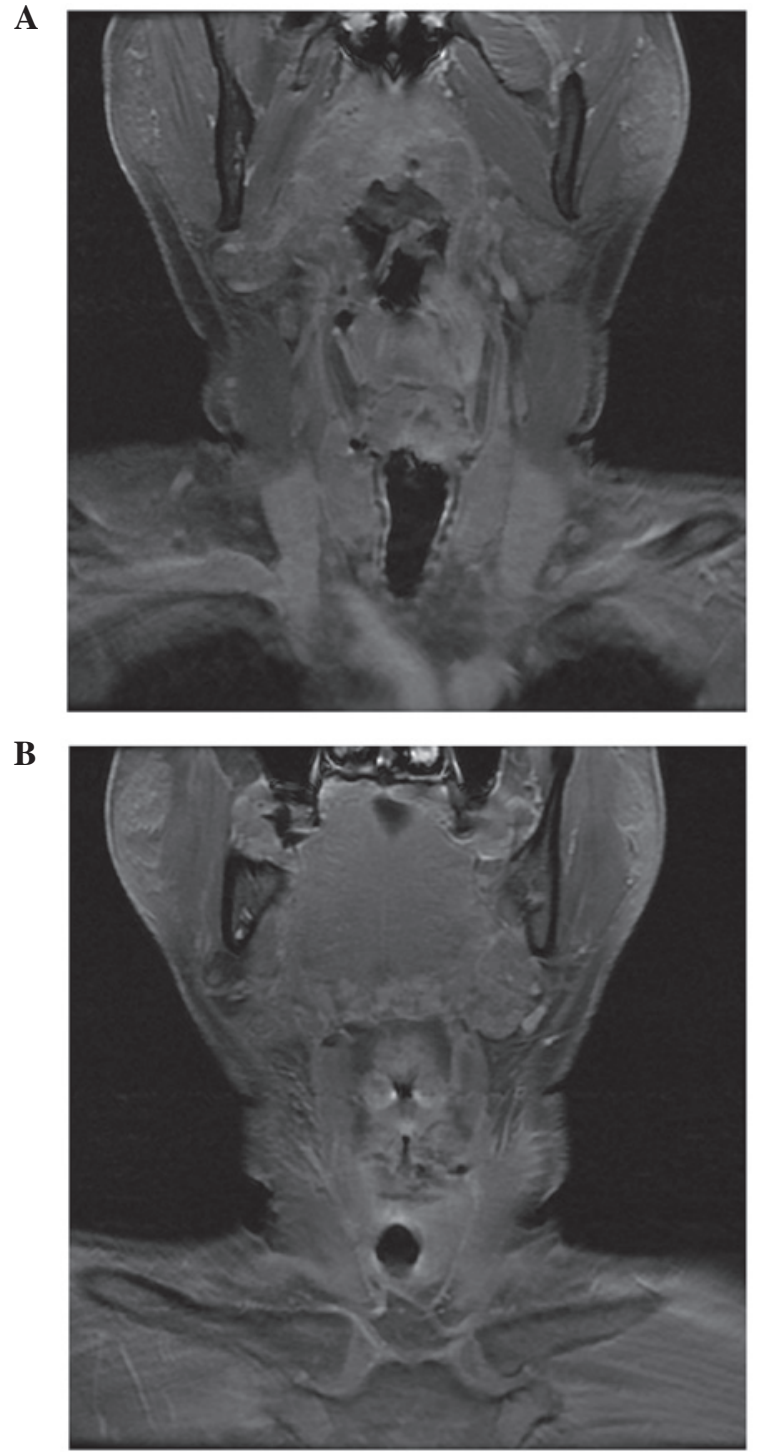

Figure 1. Magnetic resonance imaging scans from the patient. (A) T1-weighted imaging of the coronal section of the larynx shows the cyst infiltrating the subglottic area. (B) The upper edge of the cyst was shown to reach the submandibular gland.

No lymphadenectasis was observed in the neck, which suggested the possibility of a bleeding mass in the laryngeal pharynx. In addition, no evident abnormality was noted on the esophagogram. Immediately after admission, a tracheotomy was performed to alleviate the breathing difficulty, as well as a laryngeal neoplasm excision. From the original tracheal incision, the platysma myocutaneous flap was lifted and the larynx was exposed. Subglottic stenosis was identified through the incision of the cricothyroid membrane, and the tumor was found to originate from the cricoid cartilage. In addition, the tumor exhibited a spherical shape, measuring $2 \mathrm{~cm}$ by $2 \mathrm{~cm}$ in size, and was cystic-solid. The hydatid fluid from the right side of the larynx was clear, while in the left side, the fluid was serosanguinous. Microscopically, the cyst was shown to contain vessels and cavities that were full of red blood cells (Fig. 3A). Furthermore, the cyst was revealed to be separated by a fibrous septum that was formed from giant and mononuclear cells, fibroblasts, osteoclasts and osteoblasts (Fig. 3B). Thus, the pathological examination confirmed the diagnosis
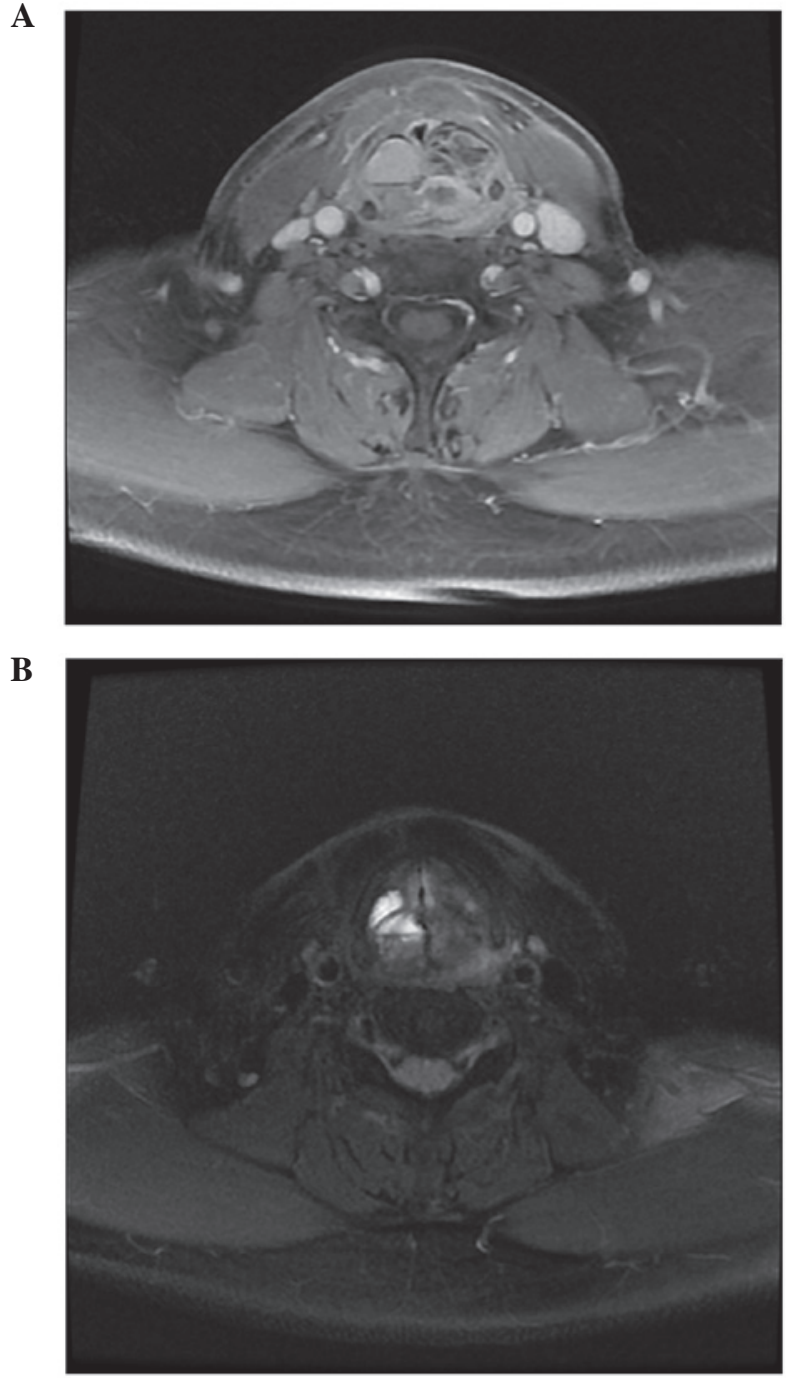

Figure 2. Magnetic resonance imaging scans from the patient. (A) T1-weighted image of the axial section of the larynx shows that the signal of the cyst is slightly higher compared with the muscle signal. (B) T2-weighted image showing the axial section of the larynx reveals an uneven cavity with a high signal, while a weak signal line was observed between the sac intervals.

of an $\mathrm{ABC}$ in the cricoid. The difficulty of the removal of the tracheal cannula appeared following resection of the hypoglottis. The patient had difficulty in breathing when he tried to block the tracheotomy tube, although he could speak almost normally by blocking the tracheal cannula for a short period of time. According to the follow-up data, no recurrence of $A B C$ was observed in this patient.

\section{Discussion}

An $\mathrm{ABC}$ is a type of osteolytic expansive cystic lesion; however, the causative factors of the tumor remain unclear. Jaffe and Lichtenstein (2) described the first case of an ABC in 1942. Jaffe hypothesized that an ABC may be the result of internal bleeding caused by other diseases; therefore, $\mathrm{ABCs}$ should be categorized as a secondary effect. By contrast, Lichtenstein proposed that $\mathrm{ABCs}$ developed from a local blood circulation dysfunction, and subsequently should be considered as a primary disorder. However, since a number 
A

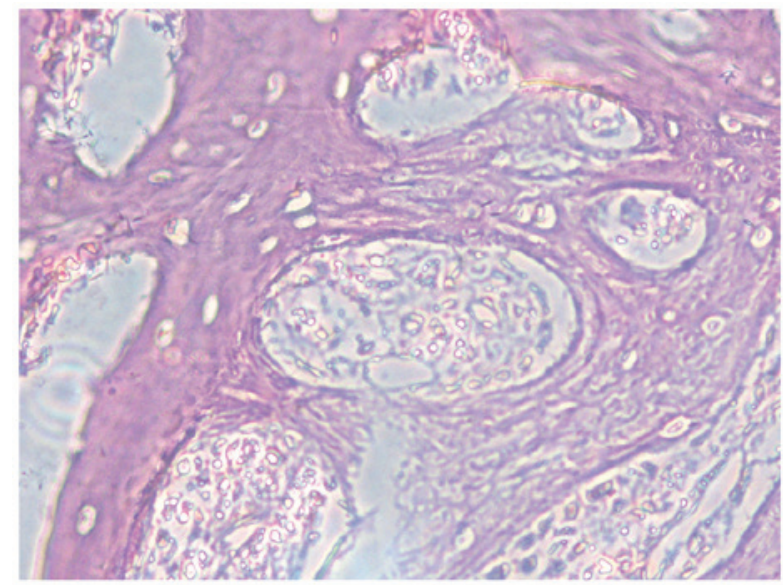

B

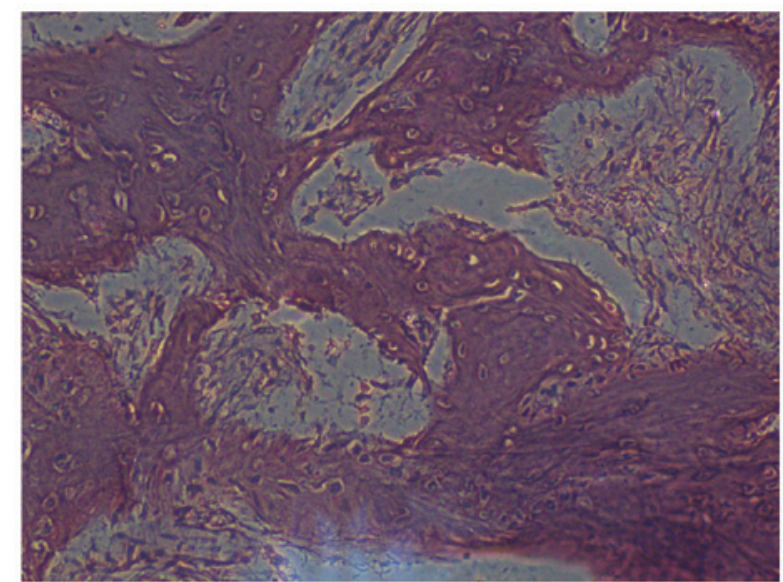

Figure 3. Histopathological specimens of the laryngeal mass. (A) Large vessels and cavities containing red blood cells can be observed (hematoxylin and eosin stain; original magnification, x20). (B) A feature of the mass formed from giant cells, mononuclear cells, fibroblasts, osteoclasts and osteoblasts (hematoxylin and eosin stain; original magnification, x10).

of vascular tumors have a tendency to form empty cavities, an increasing number of bone pathologists consider that an $\mathrm{ABC}$ is a secondary vascular change caused by a primary lesion $(2,4,5)$. During the degradation process of the lesion, an abnormal intralesional connection of the artery and vein is formed as a result of the trauma and other irritants, which triggers reactive bone changes, namely $\mathrm{ABC}$, resulting from the hemodynamic response (1). The tensile forces exerted by the laryngeal skeletal muscles, particularly the vocalis muscles, on the local perichondral or periosteal surfaces may contribute to focal changes in the ossified cartilage, predisposing the cartilage to tissue turnover and secondary neovascularization (6). Additional studies have also hypothesized that ABCs are associated with genetic alterations (7-9). From analyzing the observations of previously reported cases, the origin of a laryngeal $\mathrm{ABC}$ may also be associated with the extent of cartilage ossification $(10,11)$. Classic microscopic features of an ABC include large vessels, as well as cavities full of uncoagulated blood, separated by a fibrous septum. The large cavities contain fibroblasts, osteoclasts, giant cells, reactive woven bone (12), and focal or diffuse accumulations of hemosiderin or reactive foam cells. In addition, a certain extent of bone or bone-like fragments may be found within the fibrous septum. The cyst has a tough texture, while the inner surface is smooth, and the bursal lumen is white or rusty. A thin shell of reactive bone is located outside the cyst wall (13-15). The initial lesion can be located in $\sim 1 / 3$ of the cases $(16,17)$. The most commonly observed lesions include giant cell tumors, neuroblastoma bones, cartilage neuroblastomas, fibrous dysplasias and giant cell reparative granulomas $(18,19)$.

$\mathrm{ABCs}$ are most common in children and teenagers, and patients with the primary disease are usually aged $<20$ years $(3,20)$. The incidence of ABCs is slightly higher in females than in males, while the overall difference between men and women is not significant (3). An ABC is commonly observed at the metaphysis of a long tubular bone, although in certain cases, the cyst may be identified in short tubular bone, flat bone, the nasal cavity or sinuses (21). The type of case reported in the present study is rare, since the ABC occurred in the laryngeal cartilage ring. According to available data, only four such cases, with no gender or age tendencies, have been published in English medical literature (10).

$\mathrm{ABCs}$ are known to exhibit unique imaging properties. In computed tomography images of an $\mathrm{ABC}$, the previous lesions can be observed, in addition to a visible aspect of cortical bone pressure trace and a thick bone crest (16). By contrast, MRI shows the clear boundary of the lobulated mass (22) and a characteristic liquid-liquid surface, particularly on T1-weighted images. In the present case, the signal intensity on the T1-weighted image was slightly higher compared with the muscle signal, while the T2-weighted image revealed an uneven cavity with a high signal, and a weak signal line between the sac intervals. Diagnosing with imageology, particularly with MRI, is significant for the diagnosis and differential diagnosis of this condition. A previous study suggested that MRI analysis results in a longer period of examination and higher resolution images of the organization; therefore, MRI may allow for the high-density cells inside the lesions to subside, which may subsequently allow for an improved display of the fluid-fluid levels (23). The imaging observations of the present case were characteristic of an ABC.

Presumably, the symptoms of an laryngeal ABC should have a certain association with the tumor size. In addition, a variety of symptoms, such as breathing difficulty, hoarseness and local bleeding, are hypothesized to be primarily caused by the compression of local tissues. With consideration of the current case, the most common symptom was breathing difficulty. Thus, the unremarkable clinical symptoms are of limited usefulness for diagnosis.

The main treatment methods for ABCs include excision, curettage and hormone injection therapy. Currently, the primary therapeutic strategy is curettage; however, this approach has a risk of recurrence. The chance of recurrence following local excision is low; however, this treatment method may cause greater damage to the patients, and there are certain limitations, such as the deformity of local bone structure, the loss of local tissue function, and disorders of the growth and development of bone $(1,24,25)$. A previous study demonstrated that hormone injection therapy in children with a primary $\mathrm{ABC}$ may also accomplish satisfactory curative effects that are comparable to that of curettage and bone grafting treatment (26). In the present case, the ABC occurred in the cricoid cartilage, and the patient was admitted to hospital primarily due to the complaint of dyspnea symp- 
toms. Thus, a tracheotomy was initially preformed in order to improve ventilation. Subsequently, the mass was excised and a portion of the annular cartilage was removed. However, the tracheal tube was unable to be removed following the resection of the hypoglottis, which may have been due to the invasion of the cricoid cartilage by the $\mathrm{ABC}$, subsequently destroying the original laryngeal cavity bracket. Therefore, the cartilage had lost the function of maintaining an unobstructed respiratory tract.

In general, the incidence of ABCs is low, while the probability of occurrence in the throat cricoid is even lower. Considering the lack of specific clinical manifestations in the present case, a confirmed diagnosis requires the combination of radiology and postoperative histopathology examinations. Since throat obstruction is commonly observed when an $\mathrm{ABC}$ arises in the throat cricoid area, prompt treatment of the dyspnea is required prior to excision or curettage. In addition, a long-term follow-up and a more reliable treatment method are necessary for improving the therapeutic outcomes of ABCs.

\section{References}

1. Vergel De Dios AM, Bond JR, Shives TC, et al: Aneurysmal bone cyst. A clinicopathologic study of 238 cases. Cancer 69: 2921-2931, 1992.

2. Jaffe HL and Lichtenstein L: Solitary unicameral bone cyst with emphasis on the roentgen picture, the pathologic appearance and the pathogenesis. Arch Surg 44: 1004-1025, 1942.

3. Leithner A, Windhager R, Lang S, et al: Aneurysmal bone cyst: A population based epidemiologic study and literature review. Clin Orthop Relat Res 363: 176-179, 1999.

4. Kershisnik M and Batsakis JG: Aneurysmal bone cysts of the jaws. Ann Otol Rhinol Laryngol 103: 164-165, 1994.

5. Wang VY, Deviren V and Ames CP: Reconstruction of C-1 lateral mass with titanium mesh cage after resection of an aneurismal bone cyst of the atlas. J Neurosurg Spine 10: 117-121, 2009.

6. Schilling HE,Neal GD,Nathan M and Aufdemorte TB: Aneurysmal bone cyst of the larynx. Am J Otolaryngol 7: 370-374, 1986.

7. Winnepenninckx V, Debiec-Rychter M, Jorissen M, et al: Aneurysmal bone cyst of the nose with $17 \mathrm{p} 13$ involvement. Virchows Arch 439: 636-639, 2001.

8. Oliveira AM, Hsi BL, Weremowicz S, et al: USP6 (Tre2) fusion oncogenes in aneurysmal bone cyst. Cancer Res 64: 1920-1923, 2004.

9. Ye Y, Pringle LM, Lau AW, et al: TRE17/USP6 oncogene translocated in aneurysmal bone cyst induces matrix metalloproteinase production via activation of NF-kappaB. Oncogene 29: 3619-3629, 2010.
10. Della Libera D, Redlich G, Bittesini L and Falconieri G: Aneurysmal bone cyst of the larynx presenting with hypoglottic obstruction. Arch Pathol Lab Med 125: 673-676, 2001.

11. Sercarz JA, Robert M, Alessi D, Fu YS and Calcaterra TC: Aneurysmal bone cyst of the cricoid cartilage: An unusual cause of subglottic stenosis. Head Neck 13: 457-460, 1991.

12. Iida S, Harada Y, Shimizu K, et al: Correlation between bone marrow edema and collapse of the femoral head in steroid-induced osteonecrosis. AJR Am J Roentgenol 174: 735-743, 2000.

13. Rodríguez-Peralto JL, López-Barea F, Sánchez-Herrera S and Atienza M: Primary aneurysmal cyst of soft tissues (extraosseous aneurysmal cyst). Am J Surg Pathol 18: 632-636, 1994.

14. Hao Y, Wang L, Yan M, et al: Soft tissue aneurysmal bone cyst in a 10-year-old girl. Oncol Lett 3: 545-548, 2012.

15. Nielsen GP, Fletcher CD, Smith MA, Rybak L and Rosenberg AE: Soft tissue aneurysmal bone cyst: A clinicopathologic study of five cases. Am J Surg Pathol 26: 64-69, 2002.

16. Mahnken AH, Nolte-Ernsting CC, Wildberger JE, et al: Aneurysmal bone cyst: Value of MR imaging and conventional radiography. Eur Radiol 13: 1118-1124, 2003.

17. Nguyen BD, Lugo-Olivieri CH, McCarthy EF, et al: Fibrous dysplasia with secondary aneurysmal bone cyst. Skeletal Radiol 25: 88-91, 1996.

18. Lin WC, Wu HT, Wei CJ and Chang CY: Aneurysmal bone cyst arising from fibrous dysplasia of the frontal bone (2002:2b). Eur Radiol 14: 930-932, 2004.

19. Patel PJ, Demos TC, Lomasney LM and Rapp T: Your diagnosis? Aneurismal bone cyst. Orthopedics 28: 428, 507-511, 2005.

20. Helms C: Benign cystic bone lesions. In: Fundamentals of Diagnostic Radiology. Brant WE and Helms CA (eds) 3rd edition. Lippincott Williams and Wilkins, Philadelphia, PA, pp1063-1085, 2007.

21. Szendröi M, Cser I, Kónya A and Rényi-Vámos A: Aneurysmal bone cyst: A review of 52 primary and 16 secondary cases. Arch Orthop Trauma Surg 111: 318-322, 1992.

22. Beltran J, Simon DC, Levy M, et al: Aneurysmal bone cysts: MR imaging at 1.5 T. Radiology 158: 689-690, 1986.

23. Hudson TM, Hamlin DJ and Fitzsimmons JR: Magnetic resonance imaging of fluid levels in an aneurysmal bone cyst and in anticoagulated human blood. Skeletal Radiol 13: 267-270, 1985.

24. Mankin HJ, Hornicek FJ, Ortiz-Cruz E, et al: Aneurysmal bone cyst: A review of 150 patients. J Clin Oncol 23: 6756-6762, 2005.

25. Campanacci M, Capanna R and Picci P: Unicameral and aneurismal bone cysts. Clin Orthop Relat Res 204: 25-36, 1986.

26. Cottalorda J, Kohler R, Chotel F, et al: Recurrence of aneurysmal bone cysts in young children: A multicentre study. J Pediatr Orthop B 14: 212-218, 2005. 